\title{
Expanding the class of general exact solutions for interacting two field kinks
}

\author{
A. de Souza Dutra ${ }^{a, b *}$ and A. C. Amaro de Faria Jr. ${ }^{b}$ \\ ${ }^{a}$ Abdus Salam ICTP, Strada Costiera 11, Trieste, I-34100 Italy. \\ ${ }^{b}$ UNESCO-Campus de Guaratinguetá-DFQ ${ }^{\dagger}$ \\ Departmento de Física e Química \\ 12516-410 Guaratinguetá SP Brasil
}

May 9, 2019

\begin{abstract}
In this work we extend the range of applicability of a method recently introduced where coupled first-order nonlinear equations can be put into a linear form, and consequently be solved completely. Some general consequences of the present extension are then commented.

PACS numbers: 11.27.+d, 11.30.Er
\end{abstract}

\footnotetext{
*E-mail: dutra@feg.unesp.br

${ }^{\dagger}$ Permanent Institution
} 
Despite of being less common, the systems governed by nonlinear equations are of great importance and, nowadays, there is a growing interest in dealing with such systems [1] - 15]. Unfortunately, as a consequence of the nonlinearity, in general we lose the capability of getting the complete solutions. Here we extend a demonstration [20] that is profitable for those systems in $1+1$ dimensions, whose the second-order differential equations can be reduced to the solution of corresponding first-order equations, the so called Bolgomol 'nyi-Prasad-Sommerfield (BPS) topological solitons [16. In this approach one can obtain a differential equation relating the two coupled fields which, once solved, leads to the general orbit connecting the vacua of the model. In fact, the "trial and error" methods historically arose as a consequence of the intrinsic difficulty of getting general methods of solution for nonlinear differential equations. About two decades ago, Rajaraman [17] introduced an approach of this nature for the treatment of coupled relativistic scalar field theories in $1+1$ dimensions. His procedure was model independent and could be used for the search of solutions in arbitrary coupled scalar models in $1+1$ dimensions. However, the method is limited in terms of the generality of the solutions obtained and is convenient and profitable only for some particular, but important, cases [18. Some years later, Bazeia and collaborators [19] applied the approach developed by Rajaraman to special cases where the solution of the nonlinear second-order differential equations are equivalent to the solution of corresponding first-order nonlinear coupled differential equations. By the way, Bazeia and collaborators wisely applied their solution to a variety of natural systems, since polymers up to domain walls. Last year 20], one of us showed that many of the systems studied in [19]-25] can be mapped into a first-order linear differential equation and, as a consequence, can be solved in order to get the general solution of the system. In this work we are going to show that one can extend the range of applicability of that procedure, by taking into account situations where the potential of the system can not be written in terms of a superpotential as it is usually supposed to be necessary [19-25]. After that, we trace some comments about the consequences, and possible applications, coming from these general solutions.

In order to deal with the problem, following the usual procedure to get BPS [16] solutions for nonlinear systems, one usually particularizes [19]-25] the form of the Lagrangian density

$$
L=\frac{1}{2}\left(\partial_{\mu} \phi\right)^{2}+\frac{1}{2}\left(\partial_{\mu} \chi\right)^{2}-V(\phi, \chi),
$$


by imposing that the potential must be written in terms of a superpotential $W(\phi, \chi)$ such that

$$
V(\phi, \chi)=\frac{1}{2}\left(\frac{\partial W(\phi, \chi)}{\partial \phi}\right)^{2}+\frac{1}{2}\left(\frac{\partial W(\phi, \chi)}{\partial \chi}\right)^{2}
$$

Then, the energy functional of the static configurations can be calculated straightforwardly, giving

$$
E_{B}=\frac{1}{2} \int_{-\infty}^{\infty} d x\left[\left(\frac{d \phi}{d x}\right)^{2}+\left(\frac{d \chi}{d x}\right)^{2}+W_{\phi}^{2}+W_{\chi}^{2}\right]
$$

which leads to

$$
E_{B}=\frac{1}{2} \int_{-\infty}^{\infty} d x\left[\left(\frac{d \phi}{d x}-W_{\phi}\right)^{2}+\left(\frac{d \chi}{d x}-W_{\chi}\right)^{2}+2\left(W_{\chi} \frac{d \chi}{d x}+W_{\phi} \frac{d \phi}{d x}\right)\right]
$$

and finally, considering the minimal energy field configuration, one gets

$$
E_{B}=\left|W\left(\phi_{j}, \chi_{j}\right)-W\left(\phi_{i}, \chi_{i}\right)\right|
$$

where $\phi_{i}$ and $\chi_{i}$ are the are the $i-t h$ vacuum state of the model [22].

In this case, one can easily see that solutions with minimal energy of the second-order differential equation for the static solutions in $1+1$ dimensions, can be solved through the corresponding first-order coupled nonlinear equations

$$
\frac{d \phi}{d x}=W_{\phi}(\phi, \chi), \frac{d \chi}{d x}=W_{\chi}(\phi, \chi)
$$

where $W_{\phi} \equiv \frac{\partial W}{\partial \phi}$ and $W_{\chi} \equiv \frac{\partial W}{\partial \chi}$. It is interesting to remark that the BPS solutions settle into vacuum states asymptotically. In other words, the vaccum states act as implicit boundary conditions of the BPS equations.

In this work we are going to expand the class of nonlinear models with exact solutions by relaxing the condition over the potential to a less restrictive one. Namely we uses that

$$
V(\phi, \chi)=\frac{1}{2}(A(\phi, \chi))^{2}+\frac{1}{2}(B(\phi, \chi))^{2} .
$$

As a consequence the energy functional can then be written as 


$$
E_{B}=\frac{1}{2} \int_{-\infty}^{\infty} d x\left[\left(\frac{d \phi}{d x}-A\right)^{2}+\left(\frac{d \chi}{d x}-B^{2}\right)^{2}+2\left(B \frac{d \chi}{d x}+A \frac{d \phi}{d x}\right)\right]
$$

Once again, it can be shown that the configuration which makes the energy minimal is that where the fields obey the first-order differential equations

$$
\frac{d \phi}{d x}=A(\phi, \chi), \frac{d \chi}{d x}=B(\phi, \chi)
$$

This happens because, using the above equations we see that the energy functional becomes simply

$$
E_{B}=\int_{-\infty}^{\infty} d x\left[\left(\frac{d \chi}{d x}\right)^{2}+\left(\frac{d \phi}{d x}\right)^{2}\right]
$$

which is positive definite, so rendering the minimal energy for the fields configuration obeying (9).

Furthermore, in order to guarantee that the above equations obey the correct second-order differential equations

$$
\frac{d^{2} \phi}{d x^{2}}=V_{\phi}(\phi, \chi), \frac{d^{2} \chi}{d x^{2}}=V_{\chi}(\phi, \chi)
$$

one must impose the following restriction over the, otherwise arbitrary functions,

$$
A_{\chi}(\phi, \chi)=B_{\phi}(\phi, \chi) .
$$

Apart from this constraint and presenting a computationally more involved calculation of the topological energy, this more general situation can now be studied by using, for instance the usual trial-orbit approach [19]-25] or, by a direct solution of the orbit equation as done in [20]. In this work we choose the second line of thinking. The idea is to write the following equation

$$
\frac{d \phi}{A}=d x=\frac{d \chi}{B}
$$

where the spatial differential element is a kind of invariant. So, one obtains that

$$
\frac{d \phi}{d \chi}=\frac{A(\phi, \chi)}{B(\phi, \chi)}
$$


This last equation is, in general, a nonlinear differential equation relating the scalar fields of the model. Now, if one is able to solve it completely, the function $\phi(\chi)$ can be used to eliminate one of the fields, so rendering the equations (6) uncoupled. Finally, this uncoupled first-order nonlinear equation can be solved in general, even if numerically.

At this point it is interesting to deal with a concrete example, in order to better expose the advantage of the present approach. Let us use, for instance the following functions $A$ and $B$, as the potential defining ones,

$$
\begin{aligned}
& A(\phi, \chi) \equiv \frac{\alpha_{1}}{2} \phi^{2}+\frac{\beta_{1}}{2} \chi^{2}+\gamma_{1} \phi \chi+\delta_{1} \phi+\epsilon_{1} \chi+\zeta_{1} \\
& B(\phi, \chi) \equiv \frac{\alpha_{2}}{2} \phi^{2}+\frac{\beta_{2}}{2} \chi^{2}+\gamma_{2} \phi \chi+\delta_{2} \phi+\epsilon_{2} \chi+\zeta_{2} .
\end{aligned}
$$

By requiring that the condition (12) over the $A$ and $B$ functions be kept, we get the constraints:

$$
\beta_{1}=\gamma_{2} ; \gamma_{1}=\alpha_{2} ; \epsilon_{1}=\delta_{2}
$$

If one try to construct a superpotential from the above functions $A$ and $B$, in the spirit which it is usually done, one concludes that it is not possible to do so unambiguously, predominantly due to the difference between the one-field dependent terms. For instance in the above illustrative example one would obtain the following two alternative superpotentials

$$
W_{1} \equiv \int A d \phi ; \quad W_{2} \equiv \int A d \chi
$$

leading respectively to the following first-order BPS equations

$$
\frac{d \phi}{d \chi}=\frac{A(\phi, \chi)}{\left[B(\phi, \chi)-\left(\frac{\beta_{2}}{2} \chi^{2}+\epsilon_{2} \chi+\zeta_{2}\right)\right]},
$$

and

$$
\frac{d \phi}{d \chi}=\frac{\left[A(\phi, \chi)-\left(\frac{\alpha_{1}}{2} \phi^{2}+\delta_{1} \phi+\zeta_{1}\right)\right]}{B(\phi, \chi)} .
$$

So, as we can see from the above, one can at least extend the usual BPS procedure through introduction of arbitrary functions depending on only one 
of the interacting fields. In order to have an exact explicit example, we further restrict our parameters choice by putting $\gamma_{1}=\beta_{2}=\delta_{1}=\epsilon_{1}=\epsilon_{2}=\zeta_{2}=0$, which leaves us with

$$
\frac{d \phi}{d \chi}=\frac{A(\phi, \chi)}{B(\phi, \chi)}=\frac{\frac{\alpha_{1}}{2} \phi^{2}+\frac{\beta_{1}}{2} \chi^{2}+\zeta_{1}}{\beta_{1} \phi \chi}
$$

which, after defining the field $\sigma \equiv \phi^{2}+\frac{2}{\alpha_{1}} \zeta_{1}$, leads us to the following linear differential equation [20],

$$
\frac{d \sigma}{d \chi}=\frac{\alpha_{1}}{\beta_{1} \chi} \sigma+\beta_{1} \chi
$$

and finally to the following exact orbit equation

$$
\phi^{2}=c_{0} \chi^{\frac{\alpha_{1}}{\beta_{1}}}+\frac{\beta_{1}^{2}}{2 \beta_{1}-\alpha_{1}} \chi^{2}-\frac{2 \zeta_{1}}{\alpha_{1}},
$$

with $c_{0}$ being the arbitrary integration constant. In this case the system potential is given by

$$
V(\phi, \chi)=\frac{\alpha_{1}^{2}}{8} \phi^{4}+\frac{\alpha_{1} \zeta_{1}}{2} \phi^{2}+\frac{\beta_{1}^{2}}{8} \chi^{4}+\frac{\beta_{1} \zeta_{1}}{2} \chi^{2}+\frac{\beta_{1}\left(2 \beta_{1}+\alpha_{1}\right)}{4} \phi^{2} \chi^{2}+\frac{\zeta_{i}^{2}}{2},
$$

which is to be compared here with the one discussed by Boya and Casahorran some years ago [18, and can be written, identifying their fields and ours through $\sigma \equiv \phi$ as $\rho \equiv \chi$, as

$$
V_{B C}(\phi, \chi)=\frac{1}{4} \phi^{4}-\frac{1}{2} \phi^{2}+\frac{\lambda}{4} \chi^{4}+\frac{(f-d)}{2} \chi^{2}+\frac{d}{2} \phi^{2} \chi^{2}+\frac{1}{4},
$$

from which we can map the constants of the two models in order to see, if possible, in what situation they can me mapped. From an inspection of the above potentials we conclude that $\alpha_{1}=\sqrt{2}, \zeta_{1}=-\frac{1}{\sqrt{2}}, \beta_{1}= \pm \sqrt{2 \lambda}$, and also the following restriction over the original parameters in $V_{B C}$ should be taken into account,

$$
d=\sqrt{\lambda}(2 \sqrt{\lambda} \pm 1), f=2 \lambda
$$

which implies into a constraint over the these two last parameters such that $d=f \pm \frac{\sqrt{f}}{2}$. 
At this point it is important to stress that a system like that studied in [18. can not have their solutions reached by the BPS usual procedure [19][25]. On the other hand, in their work [18] Boya and Casahorran have made a careful analysis of the soliton stability, showing under what circumnstances they are stable under linear perturbations, which now can be extended to our case, at least when the above appropriate constraint holds. Furthermore, now in a similar fashion to what has been done in the case of superpotential procedure to get topological configurations [20, we can show that there are further solutions to those obtained in 18, at least when the above mentioned restrictions are kept. Those mentioned solutions have the special property of being kink-like for both fields, in contrast with happens with the already known ones [18. An important remark at this point, is that here the solutions are by no means obtained through a trial and error approach, instead, they are exactly solved.

Let us consider now a bit more general model, where $\gamma_{1}=\beta_{2}=\delta_{1}=\epsilon_{2}=$ $\zeta_{2}=0$. By using the same field transformation than in the previous case we get the following linear differential equation

$$
\frac{d \sigma}{d \chi}=\left[\frac{\left(\alpha_{1} \sigma+\beta_{1} \chi^{2}+2 \epsilon_{1} \chi\right)}{\left(\beta_{1} \chi+\epsilon_{1}\right)}\right],
$$

which, after solved, leaves us with the exact orbit equation, connecting the two fields,

$$
\sigma(\chi)=\left[\frac{2 \epsilon_{1}^{2}+2 \alpha_{1} \epsilon_{1} \chi+\alpha_{1} \beta_{1} \chi^{2}}{\alpha_{1}\left(2 \beta_{1}-\alpha_{1}\right)}\right]+c_{0}\left(\beta_{1} \chi+\epsilon_{1}\right)^{\frac{\alpha_{1}}{\beta_{1}}},
$$

for $\alpha_{1} \neq 2 \beta_{1}$, and

$$
\sigma(\chi)=\frac{\epsilon_{1}^{2}}{2 \beta_{1}^{2}}+c_{1}\left(\epsilon_{1}+\beta_{1} \chi\right)^{2}+\frac{\left(\epsilon_{1}+\beta_{1} \chi\right)^{2}}{\beta_{1}^{2}} \ln \left(\epsilon_{1}+\beta_{1} \chi\right)
$$

for $\alpha_{1}=2 \beta_{1} ; c_{0}$ and $c_{1}$ are arbitrary integration constants. From now on, we substitute these solutions in one of the equations (9), and solve it, so obtaining a general solution for the system. In general it is not possible to solve $\chi$ in terms of $\phi$ from the above solutions, but the contrary is always granted. Here we will substitute $\phi(\chi)$ in the equation for the field $\chi$. However, for the case $\alpha_{1}=2 \beta_{1}$ it is very hard to get an exact solution and we will, as usually done in this type of situation [20] to the deal with the case where $\alpha_{1} \neq 2 \beta_{1}$. 
By doing the described substitution, one gets

$$
\frac{d \chi}{d x}= \pm\left(\beta_{1} \chi+\epsilon_{1}\right) \sqrt{\frac{2 \epsilon_{1}^{2}+2 \alpha_{1} \epsilon_{1} \chi+\alpha_{1} \beta_{1} \chi^{2}}{\alpha_{1}\left(2 \beta_{1}-\alpha_{1}\right)}+c_{0}\left(\beta_{1} \chi+\epsilon_{1}\right)^{\frac{\alpha_{1}}{\beta_{1}}}-\frac{2 \zeta_{1}}{\alpha_{1}}} .
$$

In general we can not have an explicit solution for the above equation, but one can verify numerically that the solutions are always of the same classes. Notwithstanding, some classes of solutions can be written in closed explicit forms. First of all, we should treat the system when $c_{0}=0$, because in this situation we can solve analytically the system for any value of the parameters, apart from the case where $\alpha_{1}=2 \beta_{1}$ as stated in above.

In order to get an explicit exact solution in this situation, we make a re-scaling in the spatial variable, such that $x=\beta_{1} y$, and note that starting from the Ricatti equation

$$
\frac{d \varphi}{d y}=a\left(\varphi^{2}+\frac{B^{2}}{4 A}-C\right)
$$

and making the field transformation $\varphi \equiv \sqrt{A \chi^{2}+B \chi+C}$, we finish with

$$
\frac{d \chi}{d y}=a\left(\chi+\frac{B}{2 A}\right) \sqrt{A \chi^{2}+B \chi+C} .
$$

Now, performing the following identifications,

$$
A \equiv \frac{\beta_{1}}{\left(2 \beta_{1}-\alpha_{1}\right)} ; B \equiv \frac{2 \epsilon_{1}}{\left(2 \beta_{1}-\alpha_{1}\right)} ; C \equiv \frac{2}{\alpha_{1}}\left[\frac{\epsilon_{1}^{2}}{\left(2 \beta_{1}-\alpha_{1}\right)}-\zeta_{1}\right],
$$

we are able to connect the equations (29) and (31), which allows us to obtain the solution we are seeking for, just by using the solution of the Ricatti equation (30), and substituting it in

$$
\chi_{ \pm} \equiv \frac{1}{2 A}\left[-B \pm \sqrt{B^{2}-4 A\left(C-\varphi^{2}\right)}\right], A \neq 0,
$$

or

$$
\chi \equiv \frac{\left(\varphi^{2}-C\right)}{B}, A=0, B \neq 0 .
$$

In this second hypothesis, which is not allowed for the set of parameters of the model we are considering, but which could appear in another system, we 
must take into account that the starting Ricatti equation should be written as

$$
\frac{d \varphi}{d y}= \pm 2\left(\varphi^{2}-C-\frac{\epsilon_{1} B}{\beta_{1}}\right),
$$

and performing the transformation $\varphi \equiv \sqrt{B \chi+C}$, we finish with

$$
\frac{d \chi}{d y}= \pm\left(\chi+\frac{\epsilon_{1}}{\beta_{1}}\right) \sqrt{B \chi+C} .
$$

Our final task is to obtain the solution of (30). In fact this a very well known equation but, for the sake of completeness, we map it too into a linear equation from which the solution of our original coupled nonlinear first-order differential equations will come after a sequence of mathematical manipulations. For this we perform a final transformation $\varphi \equiv \pm \sqrt{b}+\frac{1}{\Omega}$, where $b \equiv \frac{B^{2}}{4 A}-C(A \neq 0)$, leaving us with

$$
\frac{d \Omega}{d y}=a(1 \pm 2 \sqrt{b} \Omega)
$$

from whose solution we obtain

$$
\varphi(y)= \pm \sqrt{b}-\frac{2 \sqrt{b}}{ \pm 1-e^{-2 a \sqrt{b}\left(y-y_{0}\right)}},
$$

with $y_{0}$ being the arbitrary integration constant. In order to avoid a singularity in the solution, we must choose the negative sign in the above solution. After that, it is not hard to verify that the final function can be expressed as the one having the usual kink profile,

$$
\varphi(y)=\sqrt{b} \tanh \left[a \sqrt{b}\left(y-y_{0}\right)\right] .
$$

However, once the field $\varphi$ appears quadratically in the equations (33) and (34), the solution for this field will never have a kink profile. It will always have a lump behavior. This feature of the $c_{0}=0$ case was already observed in [20], and here we confirm that this behavior is still present in this more general case. Notwithstanding, we remark that if $\epsilon_{1} \neq 0$, there is a remaining possibility in this more general system, which were not present in the simpler case studied in [20]. For this case, we can look for solutions in a special situation where the $\chi$ field equation can be written in a simpler 
polynomial form. This happens provided that $B \equiv \sqrt{A C}$, and in this case one gets

$$
\frac{d \chi}{d y}=a\left(\chi+\frac{\epsilon_{1}}{\beta_{1}}\right) \sqrt{A \chi^{2}+B \chi+C}=a\left(\chi+\frac{B}{2 A}\right)(\sqrt{A} \chi+\sqrt{C}),
$$

and finally

$$
\frac{d \chi}{d y}=a \sqrt{A}\left(\chi^{\prime 2}-b^{\prime}\right)
$$

where $\chi^{\prime} \equiv \chi+\frac{1}{2}\left(\sqrt{\frac{C}{A}}+\frac{\epsilon_{1}}{\beta_{1}}\right)$ and $b^{\prime} \equiv \frac{1}{4}\left(\sqrt{\frac{C}{A}}-\frac{\epsilon_{1}}{\beta_{1}}\right)^{2}$, with the kink-like solution

$$
\chi=\sqrt{b^{\prime}} \tanh \left[a \sqrt{A b^{\prime}}\left(y-y_{0}\right)\right]-\frac{1}{2}\left(\sqrt{\frac{C}{A}}+\frac{\epsilon_{1}}{\beta_{1}}\right) .
$$

It is not difficult to conclude that this last solution will lead to what we call type B kink 20, where both interacting fields $\chi$ and $\phi$ present a kink profile, and the previous solution corresponds to that usually obtained in the literature [19],21]-25], what we call type A kink and is characterized by a lump form for the $\chi$ field and a kink form for the $\phi$ field.

As observed in [20], for the case with $c_{0} \neq 0$ we can, at least for certain relations between the potential parameters, obtain analytical exact solutions for the type B kink solution. This can be achieved through a special fixing of the arbitrary integration parameter $c_{0}$. For instance, in the case of the model on the screen in this work, we could choose $\alpha_{1}=\beta_{1}$ and, after rearranging the terms in the equation (40), conclude that the condition required in order to get a polynomial at the right-hand side of the equation for the field $\chi$, could be granted by imposing the following condition over $c_{0}$,

$$
B+c_{0} \beta_{1}=\sqrt{A\left(C+c_{0} \epsilon_{1}\right)} .
$$

A similar analysis could be done for the case where $\alpha_{1}=4 \beta_{1}$ which, despite of being more involved, lead us to the same conclusion about the possibility of generating type $\mathrm{B}$ kink through a convenient choice of $c_{0}$.

I this work we showed how to extend an approach recently introduced 20], in order to enlarge the class of nonlinear coupled field models which can have their solitonic configurations exactly solved in $1+1$ dimensions. This allowed us to, at last under certain special conditions, include models like those studied by Boya and Casahorran [18] in this class. As a bonus, we got 
a kink configuration which was not reported in that work. Furthermore, it is important to assert that the class of models which we were able to deal here could not be considered through the usual procedure, based on the idea of having a superpotential [19]-25]. Finally we should comment that similar models were very recently considered in the literature, concerning the study of Lorentz breaking models [26, 27].

Acknowledgments: The author is grateful to CNPq for partial financial support, and to Professor D. Bazeia for introducing him to this matter. This work has been finished during a visit within the Associate Scheme of the Abdus Salam ICTP. 


\section{References}

[1] E. Witten, Nucl. Phys. B 249 (1985) 557.

[2] J. Morris, Phys. Rev. D 49 (1994) 1105.

[3] J. Morris, Phys. Rev. D 51 (1995) 697.

[4] J. D. Edelstein, M. L. Trobo, F. A. Brito and D.Bazeia, Phys. Rev. D 57 (1998) 7561.

[5] G. J. Cheetham, E. J. Copeland, T.S. Evans and R. J. Rivers, Phys. Rev. D 47 (1993) 5316.

[6] G. Dvali and M. Shifman, Phys. Lett. B 396 (1997) 64; 407 (1997) $452(\mathrm{E})$.

[7] A. B. Adib and C. A. S. Almeida, Phys. Rev. E 64 (2001) 037701.

[8] D. Bazeia, Braz. J. Phys. 32 (2002) 869.

[9] M. Eto and N. Sakai, Phys. Rev. D 68 (2003) 125001.

[10] A. A. Andrianov, V. A. Andrianov, P. Giacconi and R. Soldati, J. High En. Phys. (7) (2003) Art. $N_{o} 063$.

[11] P. Sutcliffe, Phys. Rev. D 68 (2003) 085004.

[12] J. A. R. Cembranos, A. Dobado and A. L. Maroto, Phys. Rev. D 70 (2004) 096001.

[13] T. House and A. Lukas, Phys. Rev. D 71 (2005) 046006.

[14] Z. W. Chong, H. L“u and C. N. Pope, Phys. Lett. B 614 (2005) 96.

[15] C. D. Fosco and G. Torroba, Phys. Lett. B 620 (2005) 174.

[16] M. K. Prasad and C. M. Sommerfield, Phys. Rev. Lett. 35 (1975) 760;

E. B. Bolgomol 'nyi, Sov. J. Nucl. Phys. 24 (1976) 449.

[17] R. Rajaraman, Phys. Rev. Lett. 42 (1979) 200.

[18] L. J. Boya and J. Casahorran, Phys. Rev. A 39 (1989) 4298. 
[19] D. Bazeia, M. J. dos Santos and R. F. Ribeiro, Phys. Lett. A 208 (1995) 84.

[20] A. de Souza Dutra, Phys. Lett. B 626 (2005) 249.

[21] D. Bazeia and F. A. Brito, Phys. Rev. Lett. 84 (2000) 1094.

[22] D. Bazeia and F. A. Brito, Phys. Rev. D 61 (2000) 105019.

[23] D. Bazeia, R. F. Ribeiro and M. M. Santos, Phys. Rev. E 54 (1996) 2943.

[24] D. Bazeia and E. Ventura, Chem. Phys. Lett. 303 (1999) 341.

[25] D. Bazeia, V. B. P. Leite, B. H. B. Lima and F. Moraes, Chem. Phys. Lett. 340 (2001) 205.

[26] M. N. Barreto, D. Bazeia and R. Menezes, Phys. Rev. D 73 (2006) 065015.

[27] A. de Souza Dutra, M. Hot and F. A. Barone, "Two Field BPS Solutions for Generalized Lorentz Breaking Models", submitted for publication. 